\title{
Dyonic Field Equations in Arbitrary Media
}

\author{
Ila joshi and Jivan Singh Garia \\ Department of Physics, L. S. M. Govt. P.G. College, Pithoragarh- India \\ Email: ilajoshi0090@gmail.com
}

\begin{abstract}
Starting with the generalized Maxwell field equation of dyon, the matrix form of generalized Maxwell field equation is formulated in a compact manner. The Maxwell field equations and Lorentz force for dyon in term of the matrix are also derived in arbitrary media (material media). It represents an additional field, which shows polarization and magnetization for the electric and magnetic charge. Finally, the Continuity equation for dyon is also derived.
\end{abstract}

Keywords: Dyon, Electromagnetic fields, Polarization, Magnetization

\section{Introduction}

The magnetic monopole is a hypothetical elementary particle that is an isolated magnet with only one magnetic pole. The possible existence of a magnetic monopole was purposed by Dirac (1931). Dirac showed that if any magnetic monopoles exist in the universe, then all electric charge in the universe must be quantized i.e. Dirac quantization Condition. A dyon is a hypothetical particle with electric and magnetic charges. A dyon with zero electric charges is usually referred to as a magnetic monopole. Many particle theories, grand unified theories, and super string theories predict the existence of magnetic monopole and dyon. Dyons were first purposed by Julian Schwinger in 1969 as a phenomenological alternative to quarks (Schwinger 1969). The possible existence of a magnetic monopole was purposed by Dirac (1931). Extending the Dirac idea, Monopole and dyons were enhanced by the work of t'Hooft (1974) and Polyakov (1974) and extended by Julia and Zee (1975). Witten (1979) showed that monopoles are necessarily dyons. Maxwell field equations in the free space (Griffiths and Heald, 1991) are already known, have been reformulated in the case of arbitrary media (material media). The present paper deals with matrix formulation of generalized Maxwell field equations and Lorentz force for dyon. Similarly, we derived the matrix form of Maxwell field equation and Lorentz force for dyon in arbitrary media in a simple, compact and consistent manner. It has also been shown that continuity equation of dyon represents conservation of electric and magnetic charge.

\section{Maxwell Field Equations of Dyon}

Consider the existence of magnetic monopoles Dirac (1931) the generalized Maxwell equations (Barker and Graziani, 1978) defines as,

$$
\begin{aligned}
& \vec{\nabla} \cdot \vec{E}=\frac{\rho_{e}}{\varepsilon} \quad \vec{\nabla} \cdot \vec{B}=\mu \rho_{m} \\
& \vec{\nabla} \times \vec{E}=-\frac{\vec{j}_{m}}{\varepsilon}-\frac{\partial \vec{B}}{\partial t} \vec{\nabla} \times \vec{B}=\mu \vec{j}_{e}+\frac{\partial \vec{E}}{\partial t}
\end{aligned}
$$

The four Maxwell equations can be written as, 


$$
\begin{aligned}
& \vec{\nabla} \cdot\left(\begin{array}{l}
\vec{E} \\
\vec{B}
\end{array}\right)=\frac{1}{\varepsilon}\left(\begin{array}{c}
\rho_{e} \\
\rho_{m}
\end{array}\right) \\
& \vec{\nabla} \times\left(\begin{array}{l}
\vec{E} \\
\vec{B}
\end{array}\right)=\frac{1}{\varepsilon} \Omega\left(\begin{array}{l}
\vec{J}_{\vec{J}^{e}} \\
\vec{J}_{m}
\end{array}\right)+\frac{\partial}{\partial t} \Omega\left(\begin{array}{c}
\vec{E} \\
\vec{B}
\end{array}\right)
\end{aligned}
$$

Where $\Omega=\left(\begin{array}{cc}0 & -1 \\ 1 & 0\end{array}\right)$

After putting these values

$$
\rho=\left(\begin{array}{l}
\rho_{e} \\
\rho_{m}
\end{array}\right), Q=\left(\begin{array}{l}
e \\
g
\end{array}\right), \vec{j}=\left(\begin{array}{l}
\vec{j}_{e} \\
\vec{j}_{m}
\end{array}\right) \text { and } \vec{G}=\left(\begin{array}{c}
\vec{E} \\
\vec{B}
\end{array}\right)
$$

The generalized Maxwell field equations of dyon in term of matrix can be written as,

$$
\vec{\nabla} \cdot \vec{G}=\frac{\rho}{\varepsilon} \quad \vec{\nabla} \times \vec{G}=\frac{1}{\varepsilon} \Omega \vec{j}+\frac{\partial}{\partial t} \Omega \bar{G}
$$

The Lorentz force for dyon is written as,

$$
F=e(\vec{E}+v \times \vec{B})+g(\vec{B}-v \times \vec{E})
$$

Then, the Lorentz force for dyon in matrix form is derived as,

$$
F=(e \quad g)(1-v \times \Omega) \vec{G}
$$

\section{Maxwell Field Equations of Dyon in}

\section{Arbitrary Media}

Maxwell Equations of dyon in free space can be written as,

$$
\begin{aligned}
& \vec{\nabla} \cdot\left(\begin{array}{l}
\varepsilon_{0} \vec{E} \\
\frac{\vec{B}}{\mu_{0}}
\end{array}\right)=\left(\begin{array}{c}
\rho_{e} \\
\rho_{m}
\end{array}\right) \\
& \vec{\nabla} \times\left(\begin{array}{l}
\varepsilon_{0} \vec{B} \\
\frac{\vec{B}}{\mu_{0}}
\end{array}\right)=\Omega\left(\begin{array}{l}
\vec{j}_{e} \\
\vec{j}_{m}
\end{array}\right)+\frac{\partial}{\partial t} \Omega\left(\begin{array}{l}
\varepsilon_{0} \vec{B} \\
\frac{\vec{B}}{\mu_{0}}
\end{array}\right)
\end{aligned}
$$

Maxwell field Equations of dyon in arbitrary media involve the displacement field and the magnetic induction vector, for the electric and magnetic charge. Maxwell Equations of dyon in arbitrary media can be written as,

$$
\begin{aligned}
& \vec{\nabla} \cdot\left(\begin{array}{l}
\vec{D}_{e} \\
\vec{H}_{m}
\end{array}\right)=\left(\begin{array}{c}
\rho_{f e} \\
\rho_{f m}
\end{array}\right) \\
& \vec{\nabla} \times\left(\begin{array}{l}
\vec{D}_{m} \\
\vec{H}_{e}
\end{array}\right)=\Omega\left(\begin{array}{l}
\vec{j}_{f e} \\
\vec{j}_{f m}
\end{array}\right)+\frac{\partial}{\partial t} \Omega\left(\begin{array}{l}
\vec{D}_{e} \\
\vec{H}_{m}
\end{array}\right)
\end{aligned}
$$

$\rho_{f e}$ and $\rho_{f m}$ are free charge of dyon and $j_{f e}$ and $j_{f m}$ are current densities of dyon. The sources of the electromagnetic fields are electric charge and current, and magnetic charge and current, and they are divided in free and bound one for the electric and magnetic charge as,

$$
\begin{array}{ll}
\rho_{e}=\rho_{f e}+\rho_{b e} & \rho_{m}=\rho_{f m}+\rho_{b m} \\
j_{e}=j_{f e}+j_{b e} & j_{m}=j_{f m}+j_{b m} \\
\vec{D}_{e}=\varepsilon_{0} \vec{E}+\vec{P}_{e} & \vec{D}_{m}=\varepsilon_{0} \vec{E}-\vec{P}_{m} \\
\frac{\vec{B}}{\mu_{0}}-\vec{H}_{m}=-\vec{M}_{m} & \frac{\vec{B}}{\mu_{0}}-\vec{H}_{e}=\vec{M}_{e}
\end{array}
$$

Subtracting the first sets of Eq. (7) from Eq. (6) and using Eq. (8), we obtain the Maxwell Equations of dyon for $\vec{P}$ and $\vec{M}$ as,

$$
\begin{aligned}
& \vec{\nabla} \cdot-\left(\begin{array}{l}
\vec{P}_{e} \\
\vec{M}_{m}
\end{array}\right)=\left(\begin{array}{c}
\rho_{b e} \\
\rho_{b m}
\end{array}\right) \\
& \vec{\nabla} \times\left(\begin{array}{l}
\vec{P}_{m} \\
\vec{M}_{e}
\end{array}\right)=\Omega\left(\begin{array}{l}
\vec{j}_{b e} \\
\vec{j}_{b m}
\end{array}\right)-\frac{\partial}{\partial t} \Omega\left(\begin{array}{l}
\vec{P}_{e} \\
\vec{M}_{m}
\end{array}\right)
\end{aligned}
$$

The Lorentz force equation for dyon in material media involves displacement field $\vec{D}$ and the magnetic induction $\vec{H}$ vector for the electric and magnetic charge is given as,

$\vec{F}=\mu_{0} e\left(D_{m}+v \times H_{m}\right)+\mu_{0} g\left(H_{e}-v \times D_{e}\right)$

Its matrix form is derived as,

$$
\vec{F}=\mu_{0}(e \quad g)\left(\left(\begin{array}{l}
\vec{D}_{m} \\
\vec{H}_{e}
\end{array}\right)-v \times \Omega\left(\begin{array}{l}
\vec{D}_{e} \\
\vec{H}_{m}
\end{array}\right)\right)
$$




\section{Continuity Equation of Dyon}

Considering Eq.(3), the matrix form of the generalized Maxwell field Equations of dyon and by taking the time derivative for the divergence of this equation, applying the divergence to the curl of (identically zero) and solving the equations together:

$$
\begin{aligned}
& \frac{\partial}{\partial t}(\vec{\nabla} \cdot \varepsilon \vec{G})=\frac{\partial \rho}{\partial t} \\
& 0=\vec{\nabla} \cdot(\vec{\nabla} \times \vec{G})=\frac{1}{\varepsilon} \vec{\nabla} \cdot \Omega \vec{j}+\frac{\partial}{\partial t} \vec{\nabla} \cdot \Omega \vec{G} \\
& \frac{\partial \Omega \rho}{\partial t}+\vec{\nabla} \cdot \Omega \vec{j}=0
\end{aligned}
$$

After solving this equation with the help of matrix, we get

$$
\vec{\nabla} \cdot \vec{j}_{e}+\frac{\partial \rho_{e}}{\partial t}=0 \quad \vec{\nabla} \cdot \vec{j}_{m}+\frac{\partial \rho_{m}}{\partial t}=0
$$

Equation (14) represents the conservation of electric and magnetic charge.

\section{Conclusion}

Equation (3) represents the matrix formulation of generalized Maxwell's field equation of dyon. Considering polarization and magnetization, the Maxwell field equations (9) for dyons in arbitrary media are derived in matrix form. Here, the new constitute equalities with polarization and magnetization vector, bound

charge, and current density have been used for the well-known Maxwell's equations in the presence of electric \& magnetic charge. Equation (13) is the continuity equation for dyon which represents conservation of electric and magnetic charge. It is very difficult to define physical quantities including magnetic monopole. Hence symmetry between electric and magnetic charge is very useful in describing these quantities.

\section{Acknowledgements}

We sincerely thank the organizing committee for organizing the symposium and providing a better opportunity for learning and transfer of knowledge to the research community.

\section{References}

1. Barker, W.A., and Graziani, F., 1978, Am. J. Phys. 46, 1111-1115.

2. Dirac, P.A.M., 1931, Proc. Roy. Soc. (London), A133, 60.

3. Griffiths, D.J., and Heald, M.A., 1991, Am. J. Phys. 59,111-117.

4. Hooft, G't., 1974, Nuclear Phys. B79, 276-284.

5. Julia, B., and Zee, A., 1975, Phys. Rev. D 11, 2227.

6. Polyakov, A.M., 1974, ZhETF Pis. Red. 20.6, 430-433.

7. Schwinger, J., 1969, Science. 165,757761.

8. Witten, E., 1979, Phys. Lett. 86B, 283287. 